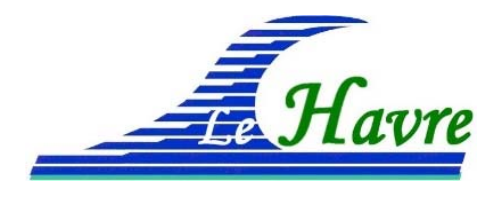

XVI èmes Journées Nationales Génie Côtier - Génie Civil

Le Havre, 2020

DOI:10.5150/jngcgc.2020.036 C Editions Paralia CFL

disponible en ligne - http://www.paralia.fr - available online

\title{
Comportement face à l'érosion côtière du trait de côte à l'Est de Cotonou
}

\section{Philippe ZOUMENOU ${ }^{1}$, Isidore DHOSSOUVI ${ }^{2}$, Marx AHLINHAN ${ }^{2}$, Yvette TANKPINOU KIKI ${ }^{2}$, Victor S. GBAGUIDI ${ }^{1}$}

\section{Université d'Abomey-Calavi, Bénin.}

2. Université Nationale des Sciences, Technologies, Ingénierie et Mathématiques, Cotonou, Bénin.

yvettetankpinou@yahoo.fr

\section{Résumé :}

Cotonou, ville côtière du Bénin située dans le Golfe de Guinée, dispose d'une façade maritime de $22 \mathrm{~km}$ de longueur sur l'Océan Atlantique. Suite à la construction en 1964 $\mathrm{du}$ Port de Cotonou et de ses nombreuses installations, une modification de la dynamique côtière et du transit sédimentaire, engendrant une accrétion de sable à l'amont (côté Ouest du Port) contre une érosion à l'aval est observée. Ainsi, pendant plus de cinquante (50) années, l'érosion côtière s'est accentuée sur la Côte Est de Cotonou par une avancée de la mer d'environ $12 \mathrm{~m}$ /an avec comme corollaires une menace sur les aménagements portuaires, l'engloutissement des infrastructures, la disparition du territoire, la dégradation des écosystèmes, la baisse des activités économiques, d'importantes conséquences d'ordre social, etc....

Depuis 2010, face à l'ampleur du phénomène aggravé par les effets néfastes des changements climatiques et face aux pressions sans cesse croissantes des populations riveraines, le programme de protection du littoral contre l'érosion côtière, logé au Ministère du Cadre de Vie et du Développement Durable, s'est attelé à la mise en œuvre de mesures de protection des segments les plus vulnérables (ANTEA GROUP, 2016). Ainsi, différents ouvrages visant à minimiser l'impact de l'érosion côtière ont été construits sur la côte Est de Cotonou pour gérer le phénomène.

Ce travail s'est d'abord intéressé à la dérive littorale observée. Il présente ensuite les données du site de projet, principalement les données métocéaniques et bathymétriques ; S'en est suivi l'état des lieux de la côte Est de Cotonou avant la mise en place des ouvrages de protection côtière. La nouvelle dynamique du trait de côte est mise en exergue en analysant les effets des constructions défensives. Enfin, les faiblesses constatées au niveau du système actuel de protection sont identifiées.

\section{Mots-clés :}

Cotonou, Trait de côte, Ouvrages de protection, Érosion, Épi. 


\section{Thème 2 - Dynamique sédimentaire}

\section{Introduction}

La région côtière du Bénin, à l'instar de celle des pays côtiers du monde, est soumise au phénomène d'érosion. En 2008, une évaluation des dégâts enregistrés du fait de l'érosion côtière sur une période de 45 ans faisait état de 337,5 ha de terrain engloutis ; 2314 ménages déplacés ; 43 immeubles détruits; 629 bâtiments d'habitation ravagés ; 4 complexes hôteliers emportés (BAIRD \& ASSOCIATES, 2013.). Les photos ci-après illustrent les manifestations du phénomène et le comportement de la côte à l'Est de Cotonou, figure 1 .

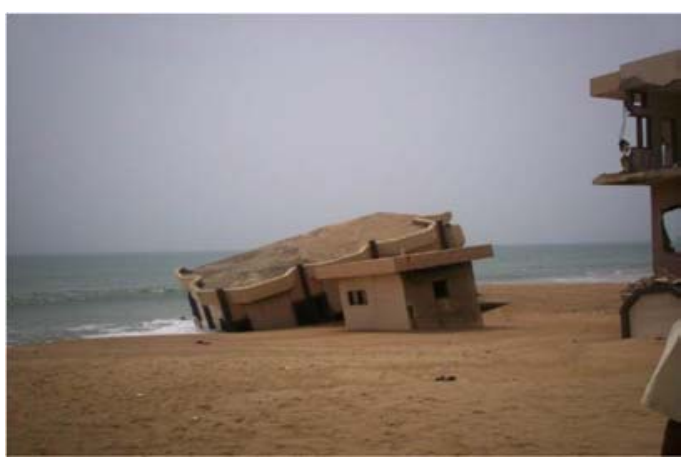

(a) Hôtel Palm Beach en disparition.

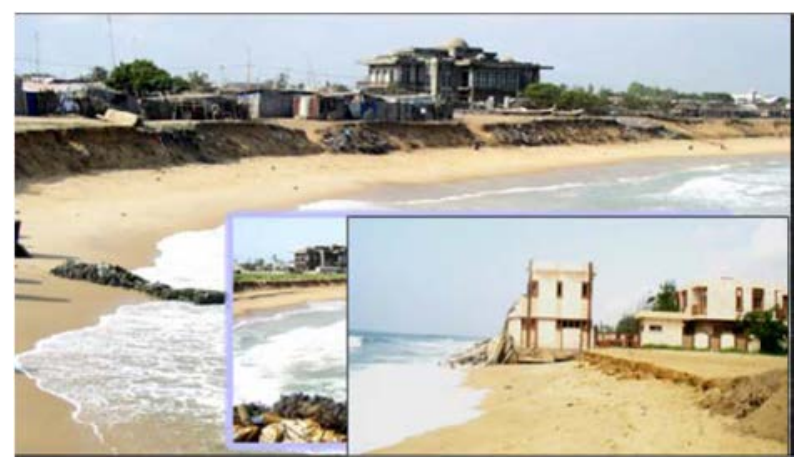

(b) route détruite et micro falaise d'érosion.

Figure 1. Manifestation de l'érosion sur le littoral de Cotonou, (ROCHE, 2015 ; DOSSOU \& GLÉHOUENOU-DOSSOU, 2007).

Dans cette situation, les riverains ont abandonné la zone, se sont constitués en organisation de défense de leurs intérêts pour exercer une pression de plus en plus croissante sur les pouvoirs publics, obligeant le gouvernement béninois à faire de la protection de cette façade maritime l'un de ses grands défis.

Ce travail a utilisé les données métocéaniques, topographiques et bathymétriques du site. L'état des lieux de la côte Est de Cotonou avant et après la mise en place des ouvrages de protection côtière a été fait. La nouvelle dynamique du trait de côte est mise en exergue en analysant les effets des constructions défensives. Enfin, les faiblesses constatées au niveau du système actuel de protection sont précisées.

\section{Milieu d'étude}

\subsection{Situation géographique}

La présente étude concerne un linéaire de $14 \mathrm{~km}$ sur la côte de Cotonou (figure 2). La zone d'étude part de l'épi d'arrêt du Port de Cotonou et s'étend jusqu'au-delà de l'épi $\mathrm{n}^{\circ}$ $7 \mathrm{du}$ système de protection côtière à l'Est de Cotonou. Ce segment comprend les infrastructures du port de Cotonou (épi d'arrêt de sable, jetée principale et jetée secondaire); la digue Ouest située au niveau du débouché du chenal de Cotonou et le champ d'épis construits sur la côte à l'Est de Cotonou. Cette présence d'ouvrages permet 


\section{XVIèmes Journées Nationales Génie Côtier - Génie Civil \\ Le Havre 2020}

de diviser le milieu d'études en trois (03) zones : la zone 1 couvre le champ d'épis de protection, la zone 2 correspond au segment situé entre le chenal de Cotonou et l'épi Est encore appelé épi de Siafato et la zone 3 qui est le segment de côte à l'Est de l'épi de fermeture du système de protection (épi numéro 7).

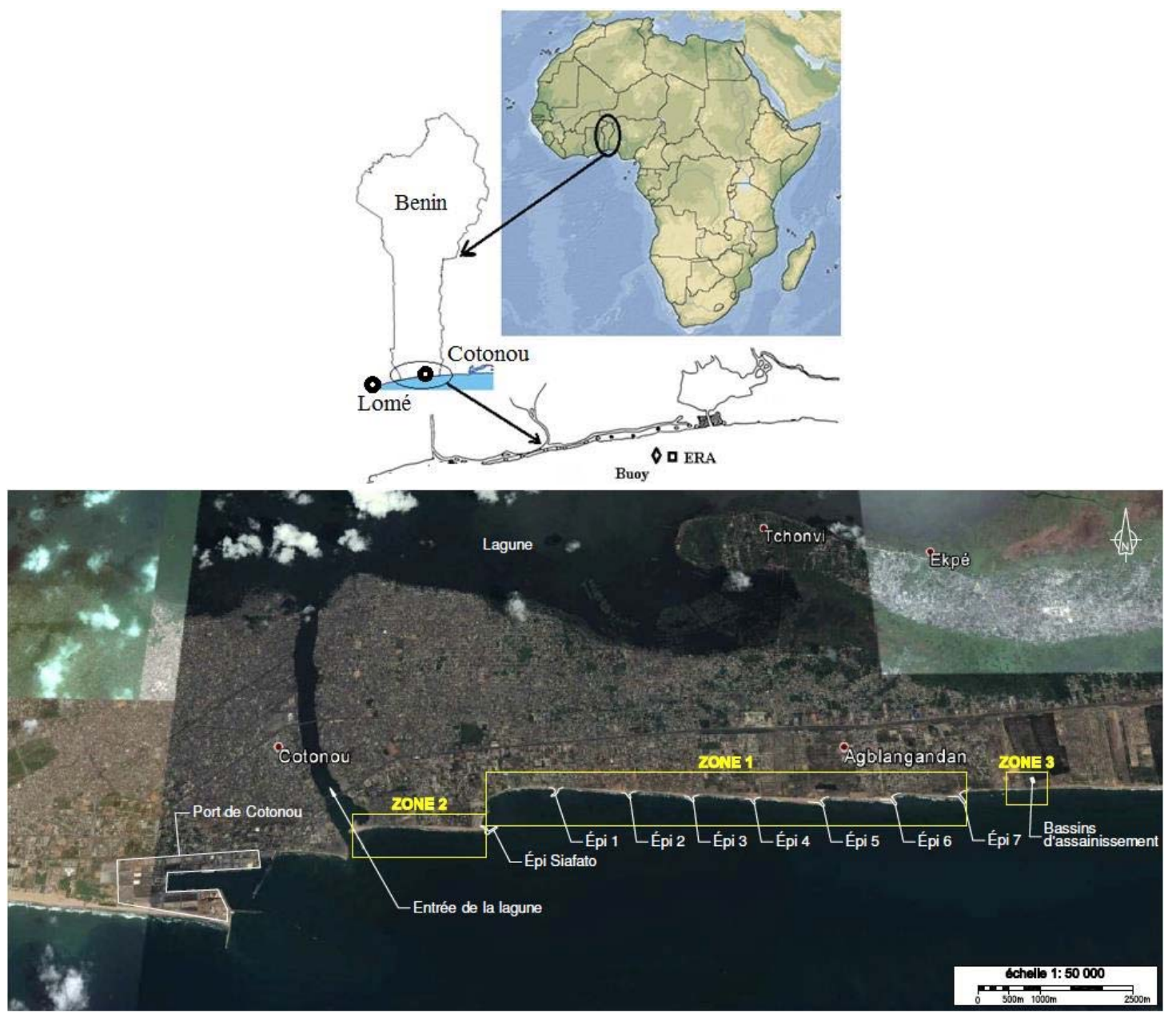

Figure 2. Situation géographique de la zone d'étude (ROCHE, 2015).

La connaissance des caractéristiques du site est essentielle pour évaluer la dynamique sédimentaire de la zone du projet et optimiser la conception des éléments constitutifs du système de protection. Les données ci-après portent sur les vents, les vagues, les niveaux d'eau, les courants (ROCHE, 2015)

\subsection{Données de vents}

Les vitesses moyennes des vents dominants varient de 10 à $30 \mathrm{~km} / \mathrm{h}(3$ à $8 \mathrm{~m} / \mathrm{s})$, avec des vitesses maximales entre les mois de juillet et septembre. Leur direction est du sud-ouest tout au long de l'année (ROCHE, 2015). Cependant, des phénomènes météorologiques locaux, tel que des orages, génèrent de forts vents sur une courte durée, typiquement en 


\section{Thème 2 - Dynamique sédimentaire}

provenance du nord et de l'est. Ces évènements extrêmes peuvent survenir à tout moment de l'année.

\subsection{Données de vagues}

La houle à Cotonou présente un caractère saisonnier très marqué : une période de faibles houles d'amplitude moyenne de 0,9 à 1,3 m centrée sur les mois de Décembre et Mars ; une période de fortes houles d'amplitude moyenne de 1,6 à 1,9 m centrée sur les mois de Juin à Août. En plus de la houle longue, il est noté l'existence des mers de vent dues à la mousson ; leur période sur le littoral béninois est de l'ordre de 6 à 8 secondes et leur amplitude dépasse rarement 1,5 mètre ; Sur les côtes béninoises, les houles viennent suivant la direction du sud au sud-ouest en général $\left(198^{\circ}\right)$. La houle a tendance à virer à l'Ouest pendant l'hivernage (juin, juillet, août, septembre).

Dans la zone de Cotonou, on observe la barre dans des profondeurs de $3 \mathrm{~m}$, soit à environ 100-200 mètres du rivage. La barre est le plus souvent constituée de plusieurs vagues successives qui déferlent et elle est le siège d'importants mouvements sédimentaires.

\subsection{Données relatives aux niveaux d'eau}

Les marées le long du littoral béninois sont de type semi-diurne avec une alternance quotidienne de deux marées basses et deux marées hautes. Le tableau 1 ci-après résume les conditions de marées le long de la côte.

Tableau 1. Tableau de marées (ROCHE, 2015).

\begin{tabular}{l|c}
\hline \multicolumn{1}{c|}{ Description } & Valeurs (mZH) \\
\hline Marée haute moyenne de vives-eaux (MHMVE) & $+1,8$ \\
Marée haute moyenne de mortes-eaux (MHMME) & $+1,0$ \\
Niveau d'eau moyen (NM) & $+0,93$ \\
Marée basse moyenne de mortes-eaux (MBMME) & $+0,40$ \\
Marée basse moyenne de vives-eaux (MBMVE) & $-0,20$ \\
\hline
\end{tabular}

\section{Dérive littorale et transport sédimentaire}

La dérive littorale est le déplacement de matières déposées par les vagues, le vent et/ou les courants longitudinaux (long shore) le long d'un littoral. Les houles obliques, les courants (de marée et autres), les vents et le déferlement causent un courant parallèle à la côte qui déplace les sédiments côtiers (sable, gravier, galets) dans une direction privilégiée.

L'effet combiné de houles régulières, d'incidence oblique et provenant du sud-ouest (incidence $5-15^{\circ}$ par rapport au trait de côte), du marnage de marée réduit, et la pente prononcée du profil de plage à caractère réflectif sont trois conditions conduisant à une dérive littorale intense dirigée vers l'est. D'après ALMAR et al. (2015), la contribution 


\section{XVIèmes Journées Nationales Génie Côtier - Génie Civil \\ Le Havre 2020}

des houles au transport total de sédiments est d'un ordre de grandeur plus élevé que le transport induit par les mers de vent, et essentiellement modulé par l'intensité et la position des vents d'ouest dans l'océan austral (30-35 $\mathrm{S}$ et 45-60 $\mathrm{S}$ ). La plupart du sable est transporté par charriage et en suspension dans une bande étroite $(30 \mathrm{~m}$ à $50 \mathrm{~m})$ entre la ligne de déferlement des vagues et le haut de plage. Les accumulations de sédiments mesurées au droit des ouvrages de défense fournissent une fourchette de valeurs comprises entre 0,4 et 1,5 $\mathrm{Mm}^{3}$ /an (TASTET et al., 1985 ; DELFT HYDRAULICS, 1990 ; ANTHONY \& BLIVI, 1999 ; BLIVI et al., 2002 ; KACZMAREK et al., 2005). Les ouvrages du Port Autonome de Cotonou interceptent à l'Ouest la quasi-totalité des sédiments du transport littoral. En se basant sur un différentiel de hauteur de profils bathymétriques datant de 2006 et 2014, ROCHE (2015) évalue le taux le volume annuel d'accrétion à 1.203.294 $\mathrm{m}^{3}$ au droit de la digue, ce qui correspond au transit sédimentaire annuel cité plus haut. Le déficit à l'est du port est relié à l'accumulation le long de la jetée ouest ; ce déficit est reparti sur deux secteurs, avec une perte qui serait de $136.000 \mathrm{~m}^{3} / \mathrm{an}$ pour la zone 2 , et $1.000 .000 \mathrm{~m}^{3} /$ an pour la zone 1 . Ainsi, la zone à 1 'ouest du port est en engraissement (figure 3) tandis que celle à l'Est est en érosion (figure 4).

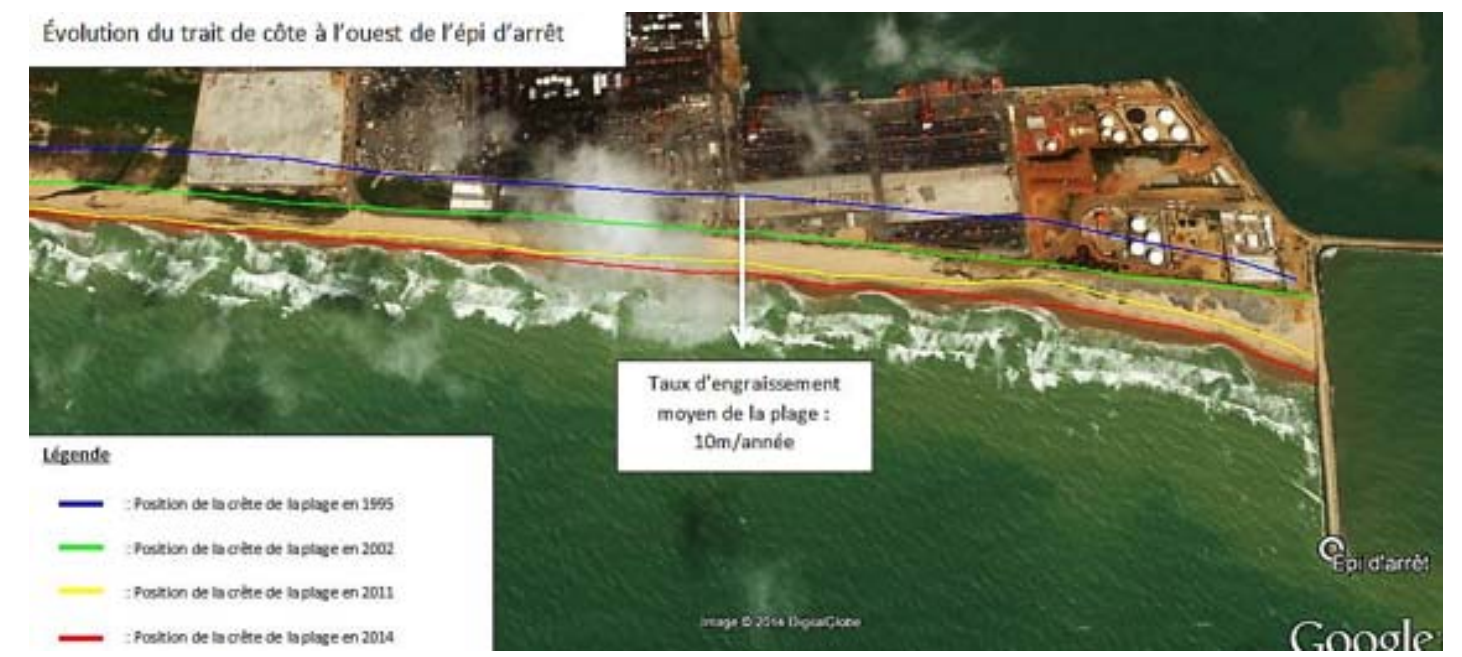

Figure 3. Evolution du trait de côte à l'Ouest de l'épi d'arrêt de sable du Port, (ROCHE, 2015). 


\section{Thème 2 - Dynamique sédimentaire}
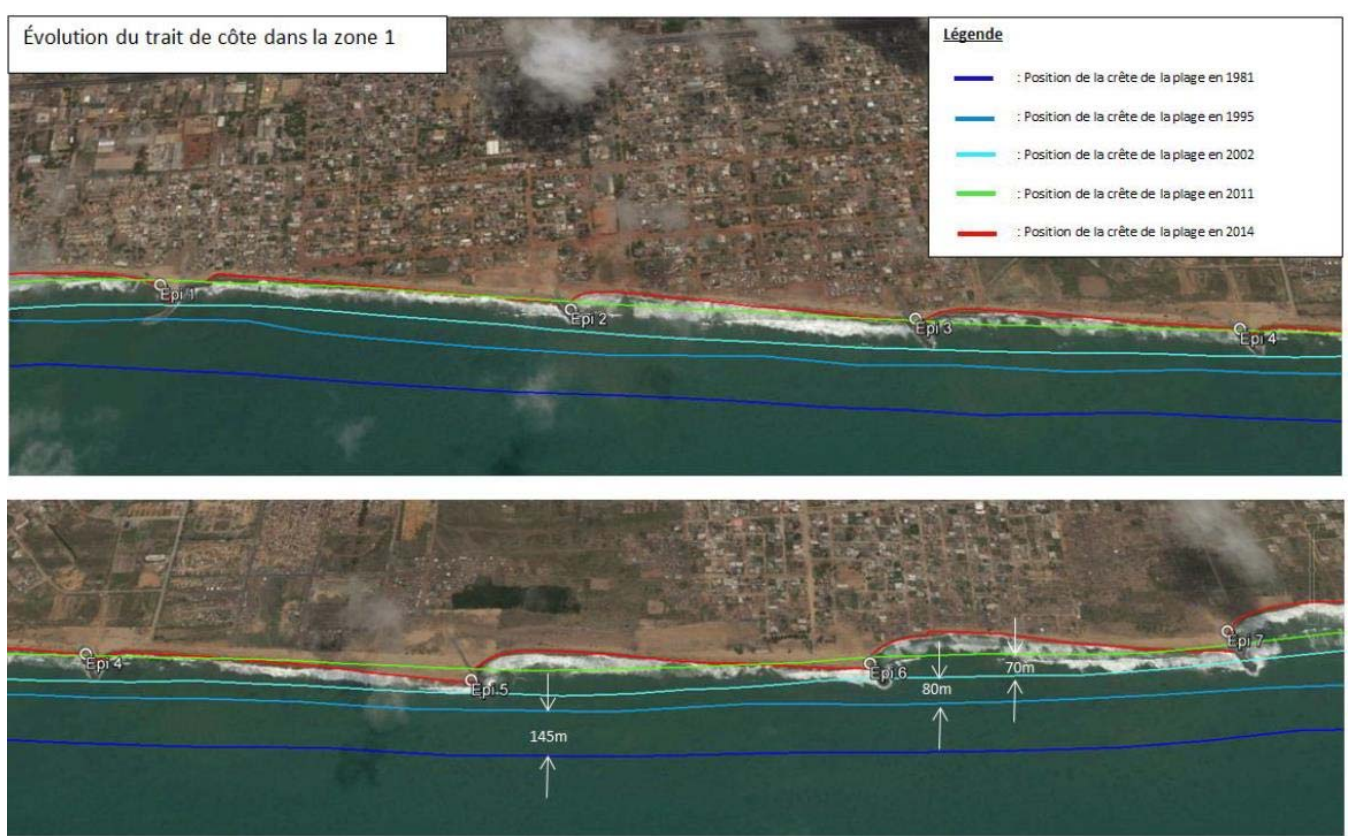

Figure 4. Evolution du trait de côte dans le champ d'épis de protection, (ROCHE, 2015).

\section{Dynamique du trait de côte}

La première étude ayant mis en évidence le retrait du trait de côte de Cotonou compare des images satellites à un intervalle de 24 ans entre 1963 et 1987, montrant un recul de $400 \mathrm{~m}$ dans la partie est de la ville, avec un taux maximal de $16 \mathrm{~m} / \mathrm{an}$ et une perte totale de 112 hectares de terrain (CODJIA et al., 1997). Une autre étude comparant des images satellites entre 1986 et 2000 a mis en évidence un retrait de la côte allant jusqu'à $150 \mathrm{~m}$ par endroits, soit un recul de plus de $10 \mathrm{~m} /$ an dans la partie Est de Cotonou, en accord avec les estimations d'autres auteurs (DOSSOU \& GLEHOUENOU-DOSSOU, 2007). ROCHE (2015) a réalisé une étude de la mobilité du trait de côte à Cotonou en se basant sur des profils de plage et des images satellites, pour en déduire les taux d'accrétion et d'érosion et évaluer la dérive littorale, figure 5. L'analyse porte sur la période 1995-2014, avant la construction des épis à l'Est du port. Le gain de surface au droit de la digue ouest du port a été de $5700000 \mathrm{~m}^{2}$ pour une perte de $6700000 \mathrm{~m}^{2}$ sur la zone située à l'est du port, le différentiel étant dû à la saturation en sable de la digue ouest et le bypass de sédiment. Si l'on compte à partir de la construction du port, en 1963, cela représente une perte d'une dizaine d'hectares de terrains par an. 


\section{XVİ̀mes Journées Nationales Génie Côtier - Génie Civil \\ Le Havre 2020}

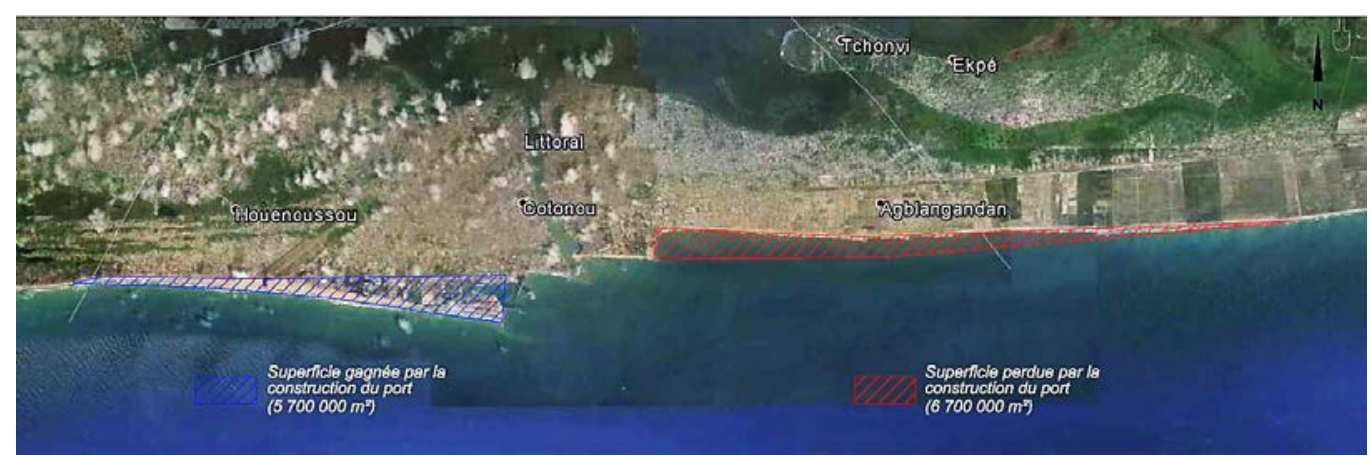

Figure 5. Bilan d'accrétion-érosion autour du Port de Cotonou, (ROCHE, 2015).

Les travaux de protection de la côte à l'Est de l'épi de Siafato, démarré en 2012 ont pris fin en 2014. La figure 6 présente la configuration de la côte à la fin des travaux.

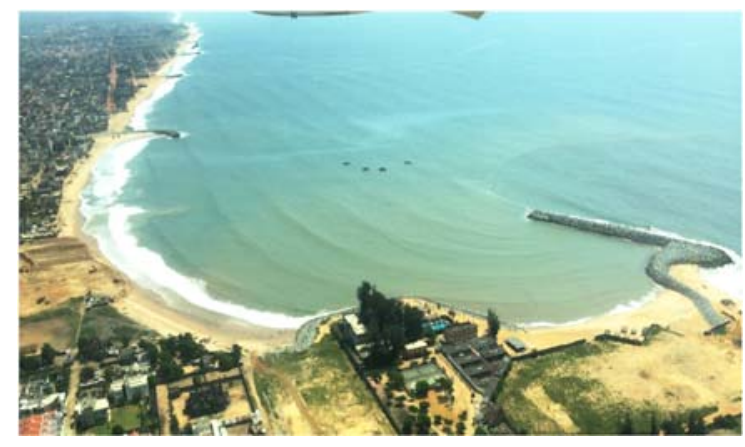

(a) Zone de la crique de Cotonou

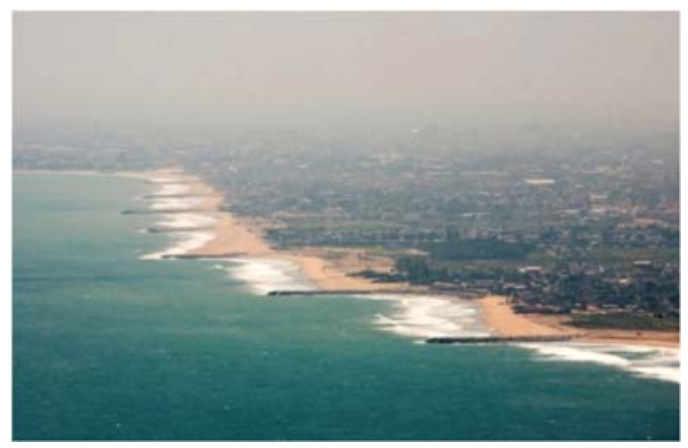

(b) champ d'épi vu de l'ouest vers l'est

Figure 6. Photographie de la configuration de la côte après la mise en place des ouvrages en 2014.

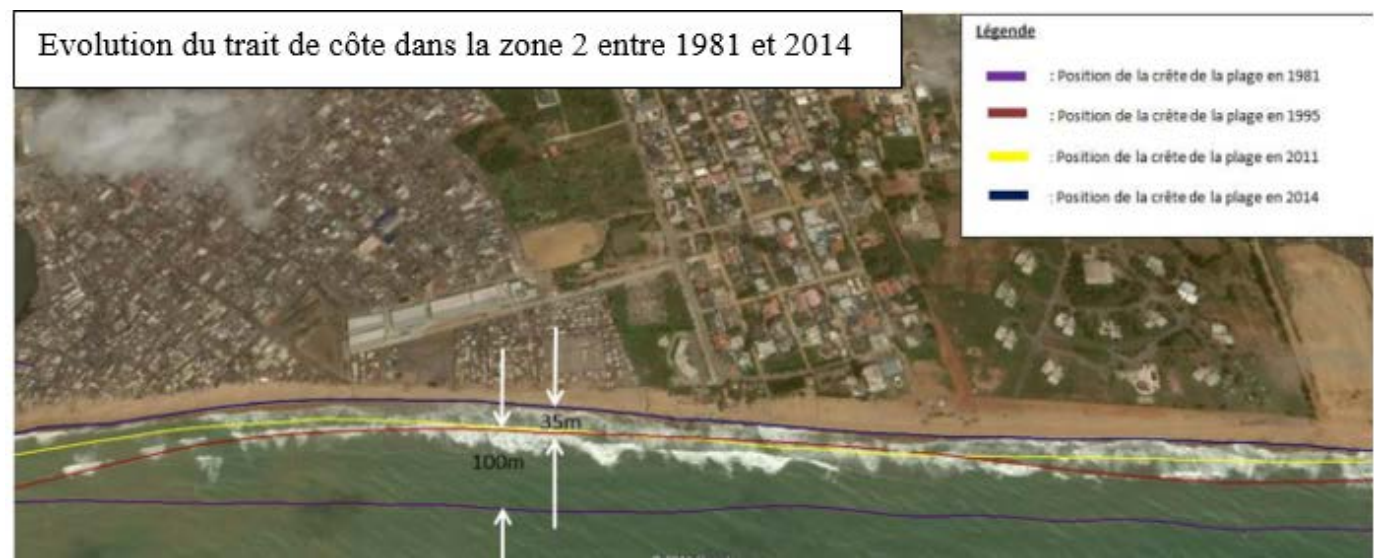

Figure 7. Evolution du trait de côte dans la zone 2, (ROCHE, 2015).

La zone 2, située entre le canal d'accès à la lagune de Cotonou et l'épi de Siafato est soumise à une érosion régulière (figure 7) avec trois périodes distinctes : une érosion importante entre 1981 et 1995 avec un recul d'une centaine de mètres au taux de $7 \mathrm{~m} / \mathrm{an}$; une relative stabilité jusqu'en 2011, et une nouvelle période érosive entre 2011 et 2014 


\section{Thème 2 - Dynamique sédimentaire}

avec un recul de $35 \mathrm{~m}$, au taux de $12 \mathrm{~m} /$ an (figure 7). L'accélération de l'érosion du trait de côte pour la période 2011-2014 pourrait être associée à l'extension de l'épi d'arrêt de sable à l'ouest du port de Cotonou dont les travaux ont été achevés en 2011. L'épi de Siafato a été reprofilé en 2012. En aval de l'épi numéro 7, implanté entre 2012 et 2014, le recul du trait de côte sur la période 1981-2014 a été de $380 \mathrm{~m}$, soit un taux de 11,5 m/an (ANGNUURENG et al., 2013).

Ainsi, la zone sous influence directe du Port présente un segment en accrétion à l'amont du Port et un segment en nette érosion à l'aval. Cependant, les segments adjacents à cette zone présentent des comportements différents: ainsi à l'Ouest, une alternance de segments en accrétion et en érosion de vitesse variant entre 0,25 et $2 \mathrm{~m} / \mathrm{an}$, est enregistrée tandis qu'à l'Est, l'alternance d'accrétion-érosion est également observée mais à une vitesse plus grande variant entre 5 et $10 \mathrm{~m} /$ an (ANTEA Group, 2016).

\section{Faiblesses constatées pour le système}

Le système de protection par les épis, développé sur la côte à l'Est de Cotonou a permis de diminuer l'érosion sur le segment abrité. Les épis construits sur la zone 1 entre 2012 et 2014 n'ont pas été rechargés initialement et n'ont pas joué pleinement leur rôle de défense contre l'érosion, entraînant des dégâts récurrents pour les zones à arrière des épis. Ainsi, malgré l'installation de ces ouvrages, l'érosion n'avait pas cessé. L'observation du système et les relevés montrent un réalignement du trait de côte dans le système d'épis mais avec de l'érosion à l'aval des ouvrages. Ceci justifie la nécessité d'injecter le sable dans le système. Cette solution mise en œuvre dans la deuxième phase du projet démarrée en 2017 et achevée en 2019 permet aujourd'hui un meilleur comportement du trait de côte et une bonne tenue des ouvrages. Dans la zone 3, un important rechargement de sable a été réalisé pour absorber l'érosion attendue sur ce segment.

Aussi, les distances entre les épis construits lors de la première phase ( $1 \mathrm{~km}$ environ) ont entrainé un effet de by pass important qui a influencé le rendement du système. Ceci a nécessité l'introduction d'épis intermédiaires dans certains segments du système lors de la phase complémentaire du projet.

\section{Conclusion}

L'étude de protection du littoral de Cotonou contre l'érosion a permis d'apprécier les caractéristiques du littoral béninois et plus particulièrement du trait de côte de Cotonou. Sur le littoral, de nombreux secteurs se trouvent en érosion, en particulier les zones 1 et 3 , avec un recul autour de $25 \mathrm{~m} /$ an pour la zone 3 .

Les caractéristiques de la houle le long du littoral de Cotonou ont permis d'évaluer le transport sédimentaire le long des profils de plage et de déduire les taux de transport annuels. Les résultats ont montré que la capacité de transport sédimentaire net à l'ouest et à l'est de la zone d'étude excédait $1000000 \mathrm{~m}^{3} / \mathrm{an}$. Les résultats ont mis en évidence une érosion modérée au centre de la zone 2 , et une tendance au réalignement des plages 


\section{XVI'èmes Journées Nationales Génie Côtier - Génie Civil \\ Le Havre 2020}

de la zone 1, qui sont légèrement éloignées de leur orientation d'équilibre, entraînant globalement une érosion régulière à l'est des épis.

À Cotonou, la dynamique littorale a largement été perturbée par la construction des ports en eau profonde, brisant l'équilibre et l'alignement du trait de côte. Les jetées ont notamment provoqué une large accrétion de sable de plusieurs centaines de mètres jusqu'à une distance de $5 \mathrm{~km}$ en amont des structures par rapport à la dérive littorale (vers l'est). En aval des structures, les secteurs en érosion s'étendent sur des distances allant jusqu'à $20 \mathrm{~km}$, compensant l'arrêt de l'apport en sédiments à l'amont. La mise en place des ouvrages de protection côtière à travers la construction de sept nouveaux épis sur environ 7 kilomètres de côte ainsi que la réhabilitation de l'ancien épi de Siafato n'ont pas encore résolu le problème de l'érosion côtière.

\section{Références bibliographiques}

ALMAR R., KESTENARE E., REYNS J., JOUANNO, J., ANTHONY E.J., LAIBI, R, HEMER M., DU PENHOAT Y.; RANASINGHE R. (2015). Response of the Bight of Benin (Gulf of Guinea, West Africa) coastline to anthropogenic and natural forcing, Part1: Wave climate variability and impacts on the longshore sediment transport. Cont. Shelf Res., Vol. 110, pp 48-59. https://doi.org/10.1016/j.csr.2015.09.020

ANGNUURENG B.D., APPEANING A.K., WIAFE G. (2013). Impact of sea defense structures on downdrift coasts: The case of Keta in Ghana. Acad. J. Environ. Sci., Vol. 1(6), pp 104-121, http://dx.doi.org/10.15413/ajes.2013.0102

ANTEA GROUP (2016). Plan d'investissement multisectoriel pour l'adaptation aux risques côtiers face aux changements climatiques au Bénin, Rapport final, Ministère du Cadre de Vie et du Développement Durable, 271 p.

ANTHONY E.J., BLIVI A.B. (1999). Morphosedimentary evolution of a delta-sourced, driftaligned sand barrier-lagoon complex, western Bight of Benin. Mar. Geol., Vol. 158, pp 161-176. https://doi.org/10.1016/S0025-3227(98)00170-4

BAIRD \& ASSOCIATES (2013). Cotonou seas defence project. Final design report, 2013.

BLIVI A., ANTHONY E.J., OYEDE L.M. (2002). Sand barrier development in the Bight of Benin, West Africa, Ocean \& Coastal Management, Vol. 45, pp 185-200. https://doi.org/10.1016/S0964-5691(02)00054-6

CODJIA C. L., DUBOIS J.-M., DONNAY J.-P., OZER A, BOIVIN F, LAVOIE A. (1997). Application de la télédétection à l'étude des changements urbains et des transformations du littoral à Cotonou (Bénin). Télédétection des milieux urbains et périurbains, AUPELF-UREF No 6, Liège, pp 299-306.

DELFT HYDRAULICS (1990). National and regional aspects of coastal erosion in the Bight of Benin. Project 6607.43.94.155, European Development Fund, Brussels.

DG-EAU (2015). Détermination des seuils et niveaux d'alerte relatifs aux risques d'élévation du niveau de la mer et d'érosion côtière au Bénin. Rapport, 182 p. 
Thème 2 - Dynamique sédimentaire

DOSSOU K., GLEHOUENOU-DOSSOU B. (2007). The vulnerability to climate change of Cotonou (Benin): the rise in sea level. Environment and Urbanization, Vol. 19(1), pp 65-79. https://doi.org/10.1177/0956247807077149

KACZMAREK L.M., OSTROWSKI R., PRUSZAK Z., ROZYNSKI G. (2005). Selected problems of sediment transport and morphodynamics of a multi-bar nearshore zone. Estuar. Coast. Shelf Sci., Vol. 62, pp 415-425. https://doi.org/10.1016/j.ecss.2004.09.006 ROCHE (2015). Projet de protection côtière à Cotonou : étude d'avant-projet détaillée (solution finale), Rapport final, Ministère de l'Urbanisme, de l'Habitat et de l'Assainissement, 146p.

TASTET J.P., CAILLON L., SIMON B. (1985). La dynamique sédimentaire littorale devant Abidjan : impact des aménagements. Contribution à la compréhension des phénomènes d'érosion et de sédimentation UNCI-PAA. 39 p. 